\title{
Computer Aided Molecular Docking Studies on Diarylsulfonylureas as Potential Anticancer Agents
}

\author{
K. L. Deepthi \\ Asst. Professor \\ Pharmaceutical Technology \\ Division \\ S.V. College of Pharmacy \\ Etcherla, Srikakulam, India
}

\author{
K. E. V. Nagoji \\ Professor \\ Pharmaceutical Analysis \\ Division \\ S.V. College of Pharmacy \\ Etcherla, Srikakulam, India
}

\author{
A. Vasudeva Rao, Ph.D \\ Assoc. Professor \\ Pharmaceutical Chemistry \\ Division \\ S.V. College of Pharmacy \\ Etcherla, Srikakulam, India
}

\begin{abstract}
Molecular docking study was performed on a series of 28 Diarylsulfonylureas LD1-LD28 as potential cyclin-dependent kinase 2 (CDK2) inhibitors. The docking technique was applied to dock a set of representative compounds within the active site region of 3PY1 using Molegro Virtual Docker v 5.0. For these compounds, the binding free energy $(\mathrm{kcal} / \mathrm{mol})$ was determined. The docking simulation clearly predicted the binding mode that is nearly similar to the crystallographic binding mode with $1.02 \mathrm{~A}^{\circ}$ RMSD. Based on the validations and hydrogen bond interactions made by $\mathrm{R}$ substituents were considered for evaluation. The results avail to understand the type of interactions that occur between diarylsulfonylureas with 3PY1 binding site region and explain the importance of $\mathrm{R}$ substitution on diarylsulfonylurea basic nucleus.
\end{abstract}

\section{Keywords}

Molecular Docking, Diarylsulfonylureas, Cyclin-dependent kinase 2 (CDK2), Molegro Virtual Docker (MVD).

\section{INTRODUCTION}

Computer aided drug design (CADD) can be done in two ways: ligand-based or structure-based. With the availability of the 3D structure of a biological target, it is feasible to use a structure-based approach to evaluate and predict the binding mode of a ligand within the active site of the receptor with docking methods [1-8]. Now it is a popular technique used for increasing the speed of drug designing process. This was made possible by the availability of many protein structures which helped in developing tools to understand the structure function relationships, automated docking and virtual screening.

Cancer is characterized by alterations in the expression of multiple genes, leading to dysregulation of the normal cellular program for cell division and cell differentiation. This results in an imbalance of cell replication and cell death that favours growth of a tumor cell population. The characteristics that delineate a malignant cancer from a benign tumor are the abilities to invade locally, to spread to regional lymph nodes, and to metastasize to distant organs in the body. At the molecular level, all cancers have several things in common, which suggests that the ultimate biochemical lesions leading to malignant transformation and progression can be produced in an unidentical pattern which is due to alterations in gene expression. In general, malignant cancers cause significant morbidity and will be lethal to the host if not treated. Exceptions to this appear to be latent, indolent cancers that may remain clinically undetectable (or in situ), allowing the host to have a standard life expectancy. Clinically, cancer appears to posses different phenotypic characteristics. As cancerous growth progresses, genetic drift in the cell population produces cell heterogeneity such as cell antigenicity, invasiveness, and as well metastatic potentials [9-12].

Cyclin-dependent kinases are the key regulators of cell-cycle transitions. In mammalian cells, Cdk2, Cdk4, Cdk6 and associated cyclins control the $\mathrm{G} 1$ to $\mathrm{S}$ phase transition. Because proper regulation of this transition is critical for an organism's survival, these protein kinases are exquisitely regulated at different mechanistic levels and in response to a large variety of intrinsic and extrinsic signals. Cyclindependent kinase 2 (CDK2) in complex with cyclins $\mathrm{E}$ and/or $\mathrm{A}$ is a key cell cycle regulator and continues to be an attractive target for the discovery of new anti-tumor agents. In particular, inhibitors of $\mathrm{CDK}-2 /$ cyclin $\mathrm{A} / \mathrm{E}$ have already progressed into clinical trials with encouraging early results $[13,14]$. The X-ray crystal structure was obtained from the Brookhaven Protein Data Bank (http://www.rcsb.org/pdb) (PDB.ID: 3PY1).

In India cancer is one of the ten leading causes of death today and advancing in rank year by year. According to the Indian Cancer Society, about 1.5 million people suffer from cancer at any point of time in India and India has the world's highest incidences of cancer of the gall bladder, mouth and lower pharynx. In view of present scenario, development of drugs with target specific predefined anticancer potential is more essential to fight against various types of cancers. Recently, the CDK2 inhibitory activity has been hypothesized to possess therapeutic potential for treatment of cancer. Thus there is a need for rapid and efficient computational methods capable of differentiating compounds with acceptable biopharmaceutical properties, e.g. solubility, lipophilicity, ionization constant etc at an early stage in the drug discovery process. In the present study, Ligand Protein Inverse Docking (LPID) stratagies were employed on set of 28 diarylsulfonylureas which earlier reported as potential cytotoxic agents. Through In Silico docking procedures different modes of interactions exhibited by these ligands will be recognized and further examined for their predicted binding energies.

\section{MATERIALS AND METHODS}

\subsection{Software Methodology}

In the present molecular docking study, software Molegro Virtual Docker (MVD) v 5.0 (www.molegro.com) along with Graphical User Interface (GUI), MVD tools was utilized to generate grid, calculate dock score and evaluate conformers. Molecular docking was performed using MolDock docking engine of software. The scoring function used by MolDock is derived from the Piecewise Linear Potential (PLP) scoring functions. The active binding site region was defined as a spherical region which encompasses all protein within $15.0 \mathrm{~A}^{\circ}$ 
of bound crystallographic ligand atom with selected coordinates of $\mathrm{X}, \mathrm{Y}$ and $\mathrm{Z}$ axes, respectively. Default settings were used for all the calculations. Docking was performed using a grid resolution of $0.30 \mathrm{~A}^{\mathrm{o}}$ and for each of the 10 independent runs; a maximum number of 1500 iterations were executed on a single population of 50 individuals. The active binding site was considered as a rigid molecule, whereas the ligands were treated as being flexible, i.e. all non-ring torsions were allowed [15].

\subsection{Molecular Modeling}

A set of 28 new diarylsulfonylureas LD1-LD28 listed in Table 1, were synthesized, characterized and which earlier reported as potential cytotoxic agents by one of the authors Dr. Vasudeva Rao Avupati et al [16]. In the present study, a set of 28 new diarylsulfonylureas LD1-LD28 were modeled by using ISIS DRAW 2.2 software.

\subsection{Ligand Preparation}

The structures of diarylsulfonylureas LD1-LD28 were converted into suitable chemical information using Chemdraw ultra v 10.0 (Cambridge software), copied to Chem3D ultra v 10.0 to create a 3D model and, finally subjected to energy minimization using molecular mechanics $\left(\mathrm{MM}_{2}\right)$. The minimization was executed until the root mean square gradient value reached a value smaller than $0.001 \mathrm{kcal} / \mathrm{mol}$. Such energy minimized structures are considered for docking and corresponding pdb files were prepared using Chem3D ultra v 10.0 integral option (save as /Protein Data Bank (pdb)) (Table 1) [17].

\subsection{Protein Selection}

The selection of protein for docking studies is based upon several factors i.e. structure should be determined by X-ray diffraction, and resolution should be between $2.0-2.5 \mathrm{~A}^{\circ}$, it should contain a co-crystallized ligand; the selected protein should not have any protein breaks in their 3D structure. However, we considered ramachandran plot statistics as the important filter for protein selection that none of the residues present in disallowed regions [18].

\subsection{Protein Preparation}

All CDK2 X-ray crystal structures were obtained from the Brookhaven Protein Data Bank (http://www.rcsb.org/pdb). Subsequent to screening for the above specific standards the resultant protein target (PDB Code: 3PY1) was selected and prepared for molecular docking simulation in such a way that all heteroatoms (i.e., nonreceptor atoms such as water, ions, etc.) were removed and Kollmann charges were assigned [19].

\subsection{Software Method Validation}

Software method validation was performed in MVD using Protein Data Bank (PDB) protein 3PY1. The x-ray crystal structure of 3PY1 complex with co-crystallized ligand was recovered from PDB. The bio active co-crystallized bound ligand was docked with in the active site region of 3PY1. The RMSD of all atoms between the two conformations is $1.02 \mathrm{~A}^{\circ}$ indicating that the parameters for docking simulation are good in reproducing X-ray crystal structure.

\subsection{Molecular Docking}

In the present investigation, we make use of a docking algorithm called MolDock. MolDock is based on a new hybrid search algorithm, called guided differential evolution. The guided differential evolution algorithm combines the differential evolution optimization technique with a cavity prediction algorithm. We used MVD because it showed higher docking accuracy than other stages of the docking products (MVD: 87\%, Glide: $82 \%$, Surflex: $75 \%$, FlexX: $58 \%$ ) in the market $[20,21]$.

Table 1. Diarylsulfonylureas LD1-LD28 with their Moldock Scores (kcal/mol) and H-bonds interactions against Cyclin-dependent kinase 2 (CDK2)<smiles>[R]C=CC(=O)c1ccccc1NC(=O)NS(=O)(=O)c1ccc(C)cc1</smiles>

\begin{tabular}{|c|c|c|c|}
\hline $\begin{array}{l}\text { Ligand } \\
\text { Code }\end{array}$ & $\begin{array}{l}\text { 'R' Group } \\
\text { Substituent }\end{array}$ & $\begin{array}{l}\text { Moldock } \\
\text { Score } \\
\text { (kcal/mol) }\end{array}$ & $\begin{array}{l}\text { No. of H- } \\
\text { Bonds / } \\
\text { H-bond } \\
\text { Interacting } \\
\text { Residues }\end{array}$ \\
\hline LD1 & $\mathrm{C}_{6} \mathrm{H}_{5}$ & -142.793 & 1/Leu 66 \\
\hline LD2 & 4- $\mathrm{MeC}_{6} \mathrm{H}_{4}$ & -148.509 & 1/Lys 134 \\
\hline LD3 & $4-\mathrm{NMe}_{2} \mathrm{C}_{6} \mathrm{H}_{4}$ & -147.976 & $1 /$ Ser 283 \\
\hline LD4 & $3-\mathrm{OMeC}_{6} \mathrm{H}_{4}$ & -154.771 & 1/Arg 509 \\
\hline LD5 & $4-\mathrm{OMeC}_{6} \mathrm{H}_{4}$ & -146.259 & $1 /$ Tyr 308 \\
\hline LD6 & 3,4-diOMeC $6 \mathrm{H}_{3}$ & -154.008 & Nil \\
\hline LD7 & 2,4-diOMeC $6 \mathrm{H}_{3}$ & -154.88 & Nil \\
\hline LD8 & $\begin{array}{l}\text { 3,4,5-tri } \\
\mathrm{OMeC}_{6} \mathrm{H}_{2}\end{array}$ & -152.219 & 1/Cys 29 \\
\hline LD9 & $2-\mathrm{OHC}_{6} \mathrm{H}_{4}$ & -144.888 & $\begin{array}{l}\text { 2/Gly } 104, \\
\text { His } 151\end{array}$ \\
\hline LD10 & $3-\mathrm{OHC}_{6} \mathrm{H}_{4}$ & -151.099 & $\begin{array}{l}\text { 2/Glu 211, } \\
\text { Asp } 274\end{array}$ \\
\hline LD11 & $4-\mathrm{OHC}_{6} \mathrm{H}_{4}$ & -147.838 & $\begin{array}{l}\text { 2/Arg 506, } \\
\text { Trp 450 }\end{array}$ \\
\hline LD12 & $3-\mathrm{OEt}, 4-\mathrm{OHC}_{6} \mathrm{H}_{3}$ & -147.356 & $\begin{array}{l}\text { 2/Glu 208, } \\
\text { Phe } 210\end{array}$ \\
\hline LD13 & $\begin{array}{l}\text { 3-OMe,4- } \\
\mathrm{OHC}_{6} \mathrm{H}_{3}\end{array}$ & -141.17 & 1/Met 74 \\
\hline LD14 & $2-\mathrm{NO}_{2} \mathrm{C}_{6} \mathrm{H}_{4}$ & -131.464 & Nil \\
\hline LD15 & $3-\mathrm{NO}_{2} \mathrm{C}_{6} \mathrm{H}_{4}$ & -148.493 & Nil \\
\hline LD16 & $5-\mathrm{OH}, 2-\mathrm{NO}_{2} \mathrm{C}_{6} \mathrm{H}_{3}$ & -148.088 & 1/Asp 274 \\
\hline LD17 & $3-\mathrm{FC}_{6} \mathrm{H}_{4}$ & -154.175 & Nil \\
\hline LD18 & $4-\mathrm{FC}_{6} \mathrm{H}_{4}$ & -151.071 & $\begin{array}{l}\text { 2/Glu 211, } \\
\text { Asp } 274\end{array}$ \\
\hline LD19 & $2-\mathrm{ClC}_{6} \mathrm{H}_{4}$ & -214.426 & $\begin{array}{l}\text { 3/Phe 146, } \\
\text { Val } 64\end{array}$ \\
\hline LD20 & $4-\mathrm{ClC}_{6} \mathrm{H}_{4}$ & -151.658 & 1/His 183 \\
\hline LD21 & 2,4- $\mathrm{diClC}_{6} \mathrm{H}_{3}$ & -141.447 & 1/Tyr 67 \\
\hline LD22 & $3-\mathrm{BrC}_{6} \mathrm{H}_{4}$ & -146.84 & 1/Glu 122 \\
\hline LD23 & 4-Allyl-OC ${ }_{6} \mathrm{H}_{4}$ & -160.316 & $\begin{array}{l}\text { 2/Lys 721, } \\
\text { Asp } 831\end{array}$ \\
\hline LD24 & Phenylethene-yl & -184.534 & 1/Arg 132 \\
\hline LD25 & Pyrrol-2-yl & -156.15 & $\begin{array}{l}\text { 2/Phe 146, } \\
\text { Val } 64\end{array}$ \\
\hline LD26 & Pyridin-3-yl & -139.734 & $\begin{array}{l}\text { 2/Ser } 148, \\
\text { Lys } 147\end{array}$ \\
\hline LD27 & Pyridin-4-yl & -141.422 & 1/Glu 73 \\
\hline LD28 & Anthracen-9-yl & -165.854 & 1/Tyr 67 \\
\hline
\end{tabular}

Molecular docking technique was employed to dock the designed diarylsulfonylureas LD1-LD28 listed in (Table 1) against CDK2 receptor 3PY1 using MVD to locate the interaction between various compounds and CDK2. MVD 
requires the receptor and ligand coordinates in either Mol2 or PDB format. Non polar hydrogen atoms were removed from the receptor file and their partial charges were added to the corresponding carbon atoms. Molecular docking was performed using MolDock docking engine of Molegro software. The binding site was defined as a spherical region which encompasses all protein atoms within $15.0 \mathrm{~A}^{\mathrm{o}}$ of bound crystallographic ligand atom (dimensions X $\left(22.34 \mathrm{~A}^{\circ}\right)$, Y ($\left.83.32 \mathrm{~A}^{\circ}\right), \mathrm{Z}\left(-22.11 \mathrm{~A}^{\circ}\right)$ axes, respectively). Default settings were used for all the calculations. Docking was performed using a grid resolution of $0.3 \mathrm{~A}^{\circ}$ and for each of the 10 independent runs; a maximum number of 1500 iterations were executed on a single population of 50 individuals.

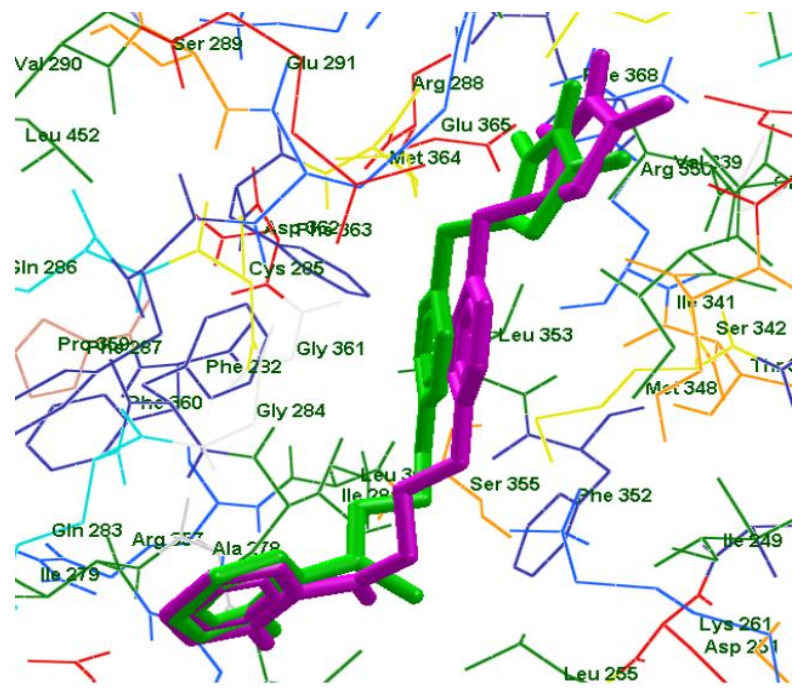

Fig 1: Superimposed binding orientation of docked conformer (pink) and most stable ligand (green) within the active binding site region of 3PY1.

\section{RESULTS AND DISCUSSION}

Ligand-Protein Inverse Docking (LPID) approach has been used as a useful tool in facilitating drug design. In this approach, docking single or multiple small molecules in single or multiple conformations to a receptor site is attempted to find putative ligands. A number of flexible docking algorithms have been introduced. These include multiple-conformer shape matching, genetic algorithm, evolutionary programming, simulated annealing, fragmentbased docking, and other novel algorithms. Testing results have shown that these algorithms are capable of finding ligands and binding conformations at a receptor site close to experimentally determined structures. Because of their capability in identifying potential ligands and binding conformations, these algorithms are expected to be equally applicable to an inverse-docking process for finding multiple putative protein targets to which a small molecule can bind or weakly bind. This may be applied to the identification of unknown and secondary therapeutic targets of drugs, drug leads, natural products and other ligands. LPID approach is now applied to the database of 28 compounds in the present study for finding possible binding orientation, binding mode and binding interaction within the active site region of CDK2. The compound with least binding energy against target protein is considered as 'hit compound'. By this means, it is possible to understand how the compounds with observed cytotoxicity interact with the target protein. The results emerging out of this study can be used to establish the possible inherent mechanism of action of diarylsulfonylureas as potential cytotoxic agents. (a)

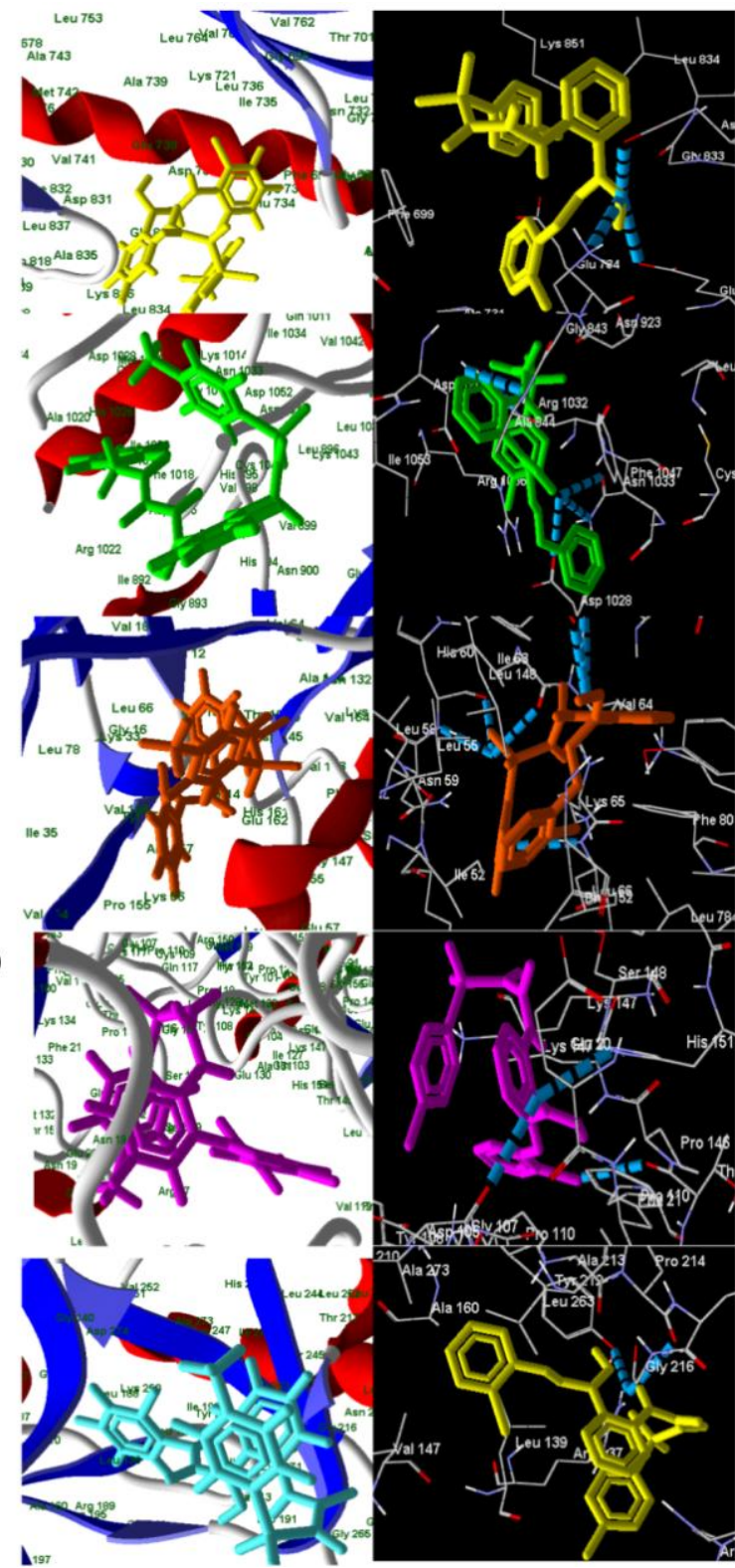

Fig 2. a) Active binding mode and H-bond interactions of LD19 against CDK-2 b) Active binding mode and H-bond interactions of LD23 against CDK-2 c) Active binding mode and H-bond interactions of LD24 against CDK-2 d)

Active binding mode and H-bond interactions of LD25

against CDK-2 e) Active binding mode and $\mathrm{H}$-bond interactions of LD28 against CDK-2

The ligand-protein inverse docking simulation technique was performed using MVD program with 28 synthetic ligands diarylsulfonylureas LD1-LD28 with basic $\alpha, \beta$-unsaturated ketone and sulfonylurea moieties reported to be having Cyclin-dependent kinase 2 (CDK2) inhibitory activity. Docking simulations with 3PY1 bound ligand resulted in a

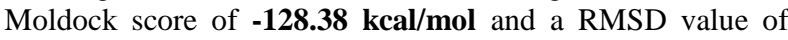
$1.72 \mathrm{~A}^{\mathbf{0}}$ showed no hydrogen bond interactions with in the active binding site region. Docking studies on experimental compounds (Table 1) showed that most stable binding ligand LD19 involved in 3 hydrogen bonds with amino acid residues Phe 146 and Val 64 within the binding site region of 3PY1. Although, other $\mathrm{H}$-bond interactions exist, these hydrogen bonds are relevant for inducing intrinsic activity towards highly selective and CDK2 specific inhibitory property. 
Moreover, from the data given in (Table 1), it appears that the compound LD19 represent most significant among the diverse range of compounds. The amino acids Phe 146 and Val 64 were appeared to be the most important binding site residues that participate in $\mathrm{H}$-bond interactions with in the active binding site region of 3PY1. The noteworthy hypothesis recognized by our studies on experimental compounds is useful in predicting the key interacting ligand LD19 and its binding properties to exhibit CDK2 specific inhibitory property. Among all the compounds with stable binding conformations as seen in case of compounds such as LD19, LD23, LD24, LD25 and LD28 and LD17 with Moldock Score i.e. least binding energies with corresponding H-bonds and interacting residues $-214.426 \mathrm{kcal} / \mathrm{mol}, 3 / \mathrm{Phe} 146$, Val 64 , $-160.316 \mathrm{kcal} / \mathrm{mol}, 2 / \mathrm{Lys} 721$, Asp $831,-184.534 \mathrm{kcal} / \mathrm{mol}$, 1/Arg 132, -156.15 kcal/mol, 2/Phe 146, Val 64 and -165.854 $\mathrm{kcal} / \mathrm{mol}, 1 /$ Tyr 67 respectively (Fig 2)

\section{CONCLUSIONS}

In this study the ligand-protein molecular docking simulation was used to preliminarily investigate and to confirm the potential molecular target for the diarylsulfonylureas LD1LD28 with observed cytotoxicity. The analysis of the best docked ligands against selected anticancer drug target revealed the binding mode of compounds involved in this study and confirm the role as CDK2 inhibitors. Binding energies of the drug-enzyme (receptor) interactions are important to describe how fit the drug binds to the target macromolecule. The residues participated in the hydrogen bond formation within the active binding site region revealed the importance of these residues towards the observed binding energy with respect to the hit identified against CDK2 target protein. The obtained hypothesis could be the remarkable starting point to develop some new leads as potential CDK2 inhibitors with enhance the affinity as well as intrinsic activity. The results of this work indicate efficient computational tools are capable of identify potential ligands such as LD19, LD23, LD24, LD25 and LD28 which earlier reported in our work as potential cytotoxic agents.

\section{ACKNOWLEDGMENTS}

One of the authors Dr. Vasudeva Rao Avupati is thankful to M/S Molegro Aps, Denmark for providing software license during the course of our research work.

\section{REFERENCES}

[1]. Drews, J. (2000) Drug discovery: A historical Perspective. Scinece 287, 1960-1964.

[2]. Ohlstein, EH., Ruffolo Jr., R.R., and Ellroff, J.D. (2000) Drug discovery in the next millennium. Annu. Rev. Pharmacol. Toxicol. 40, 177-191.

[3]. Royer RJ. Mechanism of action of adverse drug reactions: An overview. 1997; Pharmcoepidemiology and drug safety 6 (Suppl.):S43-S50.

[4]. DiMasi JA, Bryant, NR, Lasagna L. (1991). New drug development in the United States from 1963 to 1990. Clin Pharmacol Ther. 50, 471-486.

[5]. Chen Y.Z., and Zhi D. G., (2001). Ligand-Protein Inverse Docking and Its Potential Use in Computer Search of Putative Protein Targets of a Small Molecule. Proteins, 43, 217-226

[6]. Chen Y.Z., and Ung C. Y. (2002). Computer Automated Prediction of Putative Therapeutic and Toxicity Protein Targets of Bioactive Compounds from Chinese
Medicinal Plants, Am. J. Chin. Med., 30, 139-154. (2002).

[7]. Chen Y.Z., and Ung C. Y. (2001). Prediction of Potential Toxicity and Side Effect Protein Targets of a Small Molecule by a Ligand-Protein Inverse Docking Approach. J. Mol. Graph. Mod., 20, 199-218.

[8]. Cornell, WD, Cieplak P, Bayly CI, Gould IR, Merz KM Jr, Ferguson DM, Spellmeyer DC, Fox T, Caldwell JW, Kollman PA. A second generation force field for the simulation of proteins and nucleic acids. J. Am. Chem. Soc. 1995; 117:5179-5197.

[9]. Lorber DM, Shoichet BK. Flexible ligand docking using conformational ensembles. Protein Sci. 7:938-950. 1998.

[10]. Schmidt TJ, Meyer AS. Autoregulation of corticosteroid receptors. How, when, where, and why? Receptor. 1994 Winter;4:229-257.

[11].Sandak B, Wolfson HJ, Nussinov R. Flexible docking allowing induced fit in proteins: insights from an open to closed conformational isomers. Proteins 1998;32:159 174.

[12].Noreen Y, Serrano G, Perera P, Bohlin L. Flavan-3-ols isolated from some medicinal plants inhibiting COX-1 and COX-2 catalysed prostaglandin biosynthesis. Planta Med. 64:520-524. 1998.

[13].Sausville, E. A. Curr. Med. Chem. (2003), 3, 47.

[14].Fischer, P. M.; Gianella, B. A. Exp. Opin. Invest. Drugs. (2003), 12, 955.

[15].Gehlhaar, D. K.; Verkhivker, G.; Rejto, P. A.; Fogel, D. B.; Fogel, L. J.; Freer, S. T. (1995) Docking Conformationally Flexible Small Molecules Into a Protein Binding Site Through Evolutionary Programming. Proceedings of the Fourth International Conference on Evolutionary Programming, No 123-124.

[16].Vasudeva Rao Avupati, Rajendra Prasad Yejella, Girija Sankar Guntuku, Pradeepsagar Gunta (2012) Synthesis, characterization and biological evaluation of some novel diarlsulfonylureas as potential cytotoxic and antimicrobial agents. Bioorganic \& Medicinal Chemistry Letters, 22, 1031-1035.

[17].Berman H M, Westbrook J, Feng Z, Gilliland G, Bhat T N, Weissig H, Shindyalov I N, Bourne P E (2000) The Protein Data Bank. Nucleic Acids Research, 28, 235242.

[18].Wang, J, Kollman, PA, Kuntz, ID. Flexible ligand docking: A multistep strategy approach. Proteins. 1999; 36:1-19.Bowman, M., Debray, S. K., and Peterson, L. L. 1993. Reasoning about naming systems.

[19].Ramachandran, G. N., Sasisekharan, V. (1968) Conformation of polypeptides and proteins. Adv. Protein Chem. 23, 283-438.

[20].Vasudeva Rao Avupati, Purna Nagasree Kurre, Santoshi Rupa Bagadi, Muralikrishna Kumar Muthyala and Rajendra Prasad Yejella (2010) Denovo Based Ligand generation and Docking studies of PPAR $\delta$ Agonists. Correlations between Predicted Biological activity LD. Biopharmaceutical Descriptors. 10, 74-86.

[21].Storn, R., Price, K. Differential Evolution - A Simple and Efficient Adaptive Scheme for Global Optimization over Continuous Spaces. Tech-report, International Computer Science Institute, Berkley, 1995. 